\title{
Correction of iron deficiency anemia by Fe(III) hydroxocomplexes stabilized with humic ligands in rats
}

Bratishko K.A. ${ }^{1,2}$, Zykova M.V. ${ }^{1}$, Buyko E.E. ${ }^{1,2}$, Ufandeev A.A. ${ }^{1}$, Ivanov V.V. ${ }^{1}$, Perederina I.A. ${ }^{1}$, Belousov M.V. ${ }^{1,2}$, Perminova I.V. ${ }^{3}$

${ }^{1}$ Siberian State Medical University, Tomsk, Russia

${ }^{2}$ Tomsk Polytechnic University, Tomsk, Russia

${ }^{3}$ Lomonosov MSU, Department of Chemistry, Moscow, Russia

Keywords: humic ligands, iron deficiency, acute blood loss, Fe(III) hydroxocomplexes DOI:10.36291/HIT.2021.076

Iron deficiency is the most common cause of anemia. This kind of anemia (iron deficiency anemia, IDA) is usually associated with blood loss. Searching for substances with high iron bioavailability based on alternative ligands stabilizing $\mathrm{Fe}$ (III) hydroxocomplexes continues. In this regard, humic substances are promising source of new active pharmaceutical substances (APS).

C100 (Fe(III) hydroxide complex with potassium humate), C50 (Fe(III) hydroxide complex with potassium humate and polymaltose (1:1)), F100 (Fe(III) hydroxide complex with fulvic acids) and F50 (Fe(III) hydroxide complex with fulvic acids and polymaltose (1:1)) samples were used in the investigation of humic iron-containing APSs pharmacodynamic activity. A Wistar rats ( 9 ) model of acute blood loss (1.5\% of body weight) was chosen [1]. Blood was taken from the tail vein under anesthesia. The results are presented in table 1.

All studied APSs demonstrate antianemic activity. The most pronounced activity was shown for C100 sample. Less activity was observed for F100 sample. C50 and F50 showed the least activity. It can be assumed that the lowest activity of C50 and F50 is probably due to the synthesis method applicable for these APS (combined matrices). Iron disrupts polymaltose cycles, which reduces the stability of the latter.

Table 1. Influence of acute blood loss and daily (5 days) intragastric administration of $\mathrm{C} 100$, C50, F100, F50 samples and reference drug Ferrum Lek® on hematological parameters and serum iron level in rats $(\mathrm{X} \pm \mathrm{SE})$

\begin{tabular}{|c|c|c|c|c|c|c|c|c|}
\hline \multirow{2}{*}{$\begin{array}{c}\text { Group } \\
\text { of } \\
\text { animal } \\
\mathrm{s}\end{array}$} & \multicolumn{9}{|c|}{ Hematological indicators } \\
\cline { 2 - 9 } & \multicolumn{3}{|c|}{ Before IDA modeling } & \multicolumn{3}{c|}{ After course of intragastric administrations } \\
\hline $\begin{array}{c}\text { Water } \\
(\mathrm{n}=5)\end{array}$ & $193,5 \pm 6,4$ & $55,0 \pm 1,8$ & $9,6 \pm 0,6$ & $58,9 \pm 3,6$ & $147,6 \pm 2,1^{*}$ & $44,3 \pm 0,7^{*}$ & $7,2 \pm 0,1^{*}$ & $37,4 \pm 4,0^{*}$ \\
\hline $\begin{array}{c}\text { Ferrum } \\
\text { lek® } \\
(\mathrm{n}=5)\end{array}$ & $195,0 \pm 8,7$ & $59,0 \pm 2,8$ & $10,4 \pm 0,4$ & $43,9 \pm 6,3$ & $156,0 \pm 3,6^{*}$ & $47,2 \pm 4,5^{*}$ & $7,8 \pm 0,2^{*}$ & $46,5 \pm 4,5$ \\
\hline $\begin{array}{c}\text { C100 } \\
(\mathrm{n}=5)\end{array}$ & $185,0 \pm 6,4$ & $56,0 \pm 2,3$ & $10,4 \pm 0,4$ & $52,3 \pm 5,9$ & $176,4 \pm 7,7 \#$ & $52,7 \pm 2,3 \#$ & $8,8 \pm 0,4 \#$ & $50,8 \pm 2,4 \#$ \\
\hline $\begin{array}{c}\text { C50 } \\
(\mathrm{n}=5)\end{array}$ & $191,0 \pm 13,3$ & $43,6 \pm 1,6$ & $7,8 \pm 0,3$ & $56,3 \pm 7,0$ & $146,0 \pm 2,9^{*}$ & $42,6 \pm 0,8$ & $7,1 \pm 0,3$ & $38,8 \pm 7,3$ \\
\hline $\begin{array}{c}F 100 \\
(n=5)\end{array}$ & $177,0 \pm 4,7$ & $52,8 \pm 1,5$ & $9,3 \pm 0,2$ & $51,4 \pm 2,9$ & $169,6 \pm 2,0^{*} \#$ & $51,7 \pm 0,6 \#$ & $8,6 \pm 0,1 \#$ & $51,8 \pm 1,7 \#$ \\
\hline $\begin{array}{c}\text { F50 } \\
(n=5)\end{array}$ & $172,1 \pm 3,0$ & $43,5 \pm 2,8$ & $7,9 \pm 0,4$ & $58,7 \pm 3,9$ & $143,6 \pm 6,8^{*}$ & $41,4 \pm 1,9$ & $7,1 \pm 0,4$ & $51,1 \pm 4,4$ \\
\hline
\end{tabular}

Notes: 1. * differences with the group "Before IDA modeling" are statistically significant, $p<0.05$ (Friedman's test). 2. \# differences with the "Water" group are statistically significant, $p<0.05$ (KruskalWallis's criterion).

The work was carried out with the financial support of the Siberian State Medical 
University (Regulation No. 34 dated 04.09.2020).

References

1. A method for correcting post-hemorrhagic anemia // Patent of Russia No. 2017124300. 2017. / Ostroushko A.A., Tonkushina M.O., Gagarin I.D. [and etc.] 